\title{
Superresolution Microscopy of Cold Atoms in an Optical Lattice
}

\author{
Mickey McDonald, Jonathan Trisnadi, Kai-Xuan Yao, and Cheng Chin \\ James Franck Institute, Enrico Fermi Institute, and Department of Physics, \\ The University of Chicago, Chicago, Illinois 60637, USA
}

(Received 10 October 2018; revised manuscript received 14 December 2018; published 1 April 2019)

\begin{abstract}
Superresolution microscopy has revolutionized the fields of chemistry and biology by resolving features at the molecular level. In atomic physics, such a scheme can be applied to resolve the atomic density distribution beyond the diffraction limit and to perform quantum control. Here we demonstrate superresolution imaging based on the nonlinear response of atoms to an optical pumping pulse. With this technique, the atomic density distribution can be imaged with a full-width-at-half-maximum resolution of 32(4) $\mathrm{nm}$ and a localization precision below $500 \mathrm{pm}$. The short optical pumping pulse of $1.4 \mu$ s enables us to resolve fast atomic dynamics within a single lattice site. A by-product of our scheme is the emergence of moiré patterns on the atomic cloud, which we show to be immensely magnified images of the atomic density in the lattice.
\end{abstract}

DOI: 10.1103/PhysRevX.9.021001

Subject Areas: Atomic and Molecular Physics, Optics, Quantum Physics

\section{INTRODUCTION}

In the study of ultracold atomic gases, high-resolution microscopy has played an important role in visualizing interesting quantum phenomena. Examples include phase transitions [1,2], correlations [3-5], transport [6], tunneling [7], and quantum-information processing with ions [8] and atoms $[9,10]$. Optical microscopy of ultracold gases has been pushed to its limit to detect atoms in optical lattices with submicron spacings [11-17]. The spatial resolution in these experiments is constrained by the imaging wavelength to typically $0.5-1 \mu \mathrm{m}$, a value set by the Abbe limit $d=\lambda /(2 \mathrm{NA})$ [18]. Here, $\lambda$ is the wavelength of the imaging light and NA is the numerical aperture of the microscope.

Several schemes have been demonstrated which reach beyond the optical diffraction limit. Scanning electron microscopy of ultracold gases visualizes atoms with a resolution of $150 \mathrm{~nm}$ [19]. "Stochastic" techniques, which gain resolution through precise localization of point-source emitters, have been applied to determine the mean positions of trapped ions to a few nanometers [20] and the occupancy of closely spaced one-dimensional (1D) optical lattice sites [21]. Stochastic methods, however, derive their power from the assumption of

Published by the American Physical Society under the terms of the Creative Commons Attribution 4.0 International license. Further distribution of this work must maintain attribution to the author(s) and the published article's title, journal citation, and DOI. point-source emission [22], meaning that the particle distribution within a single site cannot be resolved.

Another class of superresolution imaging techniques is "deterministic," achieving genuine subwavelength resolution by exploiting the nonlinear optical response of atoms and molecules to a spatially varying light field [23,24]. For cold atoms, proposals exist based on spatially dependent coherent dark-state transfer [25-28] and have been implemented to demonstrate subwavelength addressing of a thermal atomic cloud [29].

In this paper, we demonstrate 1D superresolution microscopy of ultracold atoms at the nanometer scale. Our technique shares conceptual similarities to saturated structured illumination microscopy [24] and stimulated emission depletion microscopy [23] and is schematically illustrated in Fig. 1. Atoms are initially localized in the trapping lattice and polarized in the $|F=3\rangle$ ground state, where $F$ is the total angular momentum. An additional optical pumping (OP) lattice is applied which pumps atoms to a different hyperfine state $|F=4\rangle$. Since just a few photons are required to pump atoms to the new state, only atoms within a narrow window around the nodes of the OP lattice are likely to remain in the initial state, while those outside this window have near-unity probability to be pumped to the $|F=4\rangle$ state. By sweeping the location of this window across the atomic density distribution and measuring the fraction of atoms remaining in $|F=3\rangle$, a map of the atomic density distribution can be built up with a resolution given by the width of the window. As we discuss below, this width can be made arbitrarily small compared to the optical wavelength, which is key to attaining high resolution. 
(a)

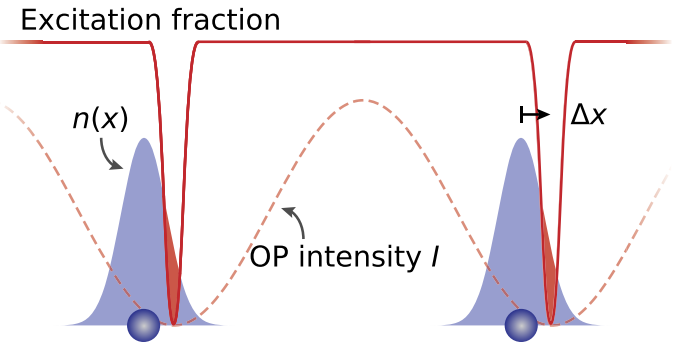

(b)

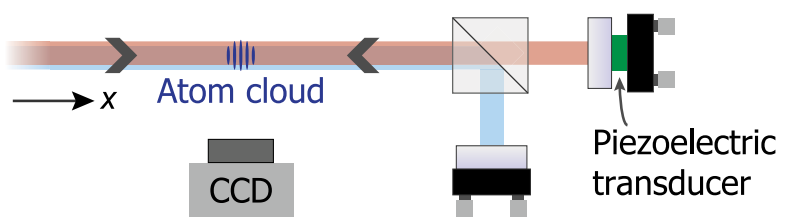

(c)

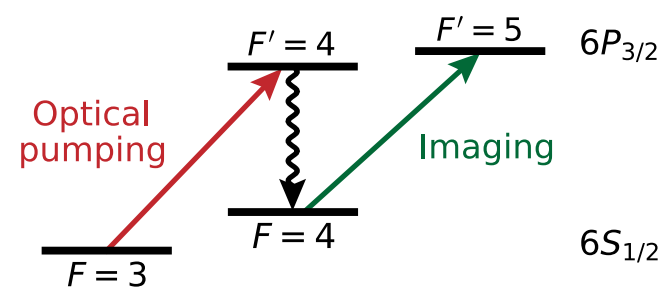

FIG. 1. Superresolution imaging of ultracold atoms based on optical pumping. (a) Given an intense standing wave of OP light, the excitation probability (red solid line) is nearly unity unless the atoms (blue shaded area) are within a narrow window at the nodes of the OP lattice (red dashed line) where the intensity vanishes. Red shaded area approximates the fraction of the atoms that are not excited. (b) The trapping lattice (cyan) and OP lattice (red) overlap on the atoms. The relative displacement between the two lattices is controlled by a piezoelectric transducer behind one of the retroreflection mirrors. The atomic distribution is measured by scanning the piezoelectric displacement $\Delta x$. A CCD camera from the side images atoms in situ on the strong $|F=4\rangle \rightarrow$ $\left|F^{\prime}=5\right\rangle$ cycling transition. (c) Relevant atomic levels. Atoms initially in the $|F=3\rangle$ ground state are optically pumped to $|F=4\rangle$ and imaged with the imaging beam. Primed and unprimed letters refer to levels within the excited-state $6^{2} P_{3 / 2}$ and ground-state $6^{2} S_{1 / 2}$ manifolds, respectively.

\section{EXPERIMENTAL SETUP}

Our experimental implementation is illustrated in Fig. 1(b). A cloud of ${ }^{133} \mathrm{Cs}$ atoms is collected in a magnetooptical trap, subsequently cooled by degenerate Ramansideband cooling to $<1 \mu \mathrm{K}$ [30], and polarized in the $|F=3\rangle$ ground state. About $2 \times 10^{6}$ atoms are then adiabatically loaded into a one-dimensional optical lattice with approximately $90 \%$ occupation in the motional ground state along the lattice direction. The trapping lattice with lattice constant $\lambda_{\text {trap }} / 2 \approx 426 \mathrm{~nm}$ is blue-detuned from the resonance transition $|F=3\rangle \rightarrow\left|F^{\prime}=4\right\rangle$ by $\delta=+10$ to $500 \mathrm{GHz}$. The OP laser is resonant with the $|F=3\rangle \rightarrow$ $\left|F^{\prime}=4\right\rangle$ transition at $\lambda_{\mathrm{OP}}=852.335 \mathrm{~nm}$ [see Fig. 1(c)] and is retroreflected and polarized perpendicularly to the copropagating trapping lattice. The retroreflection of the OP beam is carefully aligned and balanced to cancel the electric field at the nodes of the standing wave. The relative phases of the two lattices are controlled with nanometer precision using a piezoelectric transducer [31].

To image the atoms, we apply a $1.4-\mu$ s pulse of the OP lattice while the atoms are still confined to the trapping lattice, which transfers atoms to $|F=4\rangle$. This pulse is short compared to the timescale of atomic motion but much longer than the $6 P_{3 / 2}$ excited-state lifetime of $1 / \Gamma=30 \mathrm{~ns}$. The OP pulse is followed by in situ imaging with a camera in the direction perpendicular to the lattice. From measuring the atomic population in $|F=4\rangle$, we determine the excitation fraction $\mathcal{F}$ across the sample.

\section{RESULTS AND DISCUSSION}

\section{A. Determining and Characterizing Resolution}

To explore the resolving power of this technique, we record traces of the excitation fraction $\mathcal{F}$ versus piezoelectric displacement $\Delta x$ (see Fig. 2). At sufficiently low OP beam intensities $I \ll I_{\text {sat }}$, the excitation fraction $\mathcal{F}(\Delta x)$ varies sinusoidally, mirroring the sinusoidal intensity profile of the OP lattice $I(x)=4 I \sin ^{2}\left(2 \pi x / \lambda_{\mathrm{OP}}\right)$. At higher intensities, however, the response of atoms to optical pumping becomes more nonlinear because the excitation fraction quickly saturates to 1 unless atoms are located sufficiently close to the nodes of the OP lattice. In this regime, the remaining fraction $g(x)$ of atoms in the $|F=3\rangle$ state near a node is approximately given by

$$
g(x)=\exp \left(-\frac{\beta \Gamma}{2} \frac{I(x)}{I(x)+I_{\mathrm{sat}}} t\right)
$$

where we assume a long exposure time $t \gg 1 / \Gamma$, and $\beta=7 / 12$ is the branching ratio of spontaneous emission into the $|F=4\rangle$ state. At the nodes, $g(x)$ develops narrow peaks.

The narrowing of the excitation dips at higher OP intensity [see Fig. 2(b)] results from the nonlinear optical response described in Eq. (1). This narrowing can also be understood as revealing the atomic density distribution with increasing resolving power. Given a spatial density distribution $n(x)$ for an atom (in either a pure or mixed quantum state) under the spatially varying OP intensity $I(x)$, the excitation fraction $\mathcal{F}(\Delta x)$ directly relates to the atomic density $n(x)$ as

$$
1-\mathcal{F}(\Delta x)=\int n(x) g(\Delta x-x) d x \equiv \tilde{n}(\Delta x),
$$

where $\tilde{n}(\Delta x)$ is the convolution of the atomic density distribution with the point-spread function given by $g(x)$. When the width of $g(x)$ is smaller than that of the atomic density distribution, $\tilde{n}(\Delta x)$ (and, equivalently, $1-\mathcal{F}$ ) reveals the atomic density distribution [see Figs. 2(c) and 2(d)]. Because the excitation fraction $\mathcal{F}$ is measured with a finite imaging resolution, the extracted density 
(a)

(b)



(d)

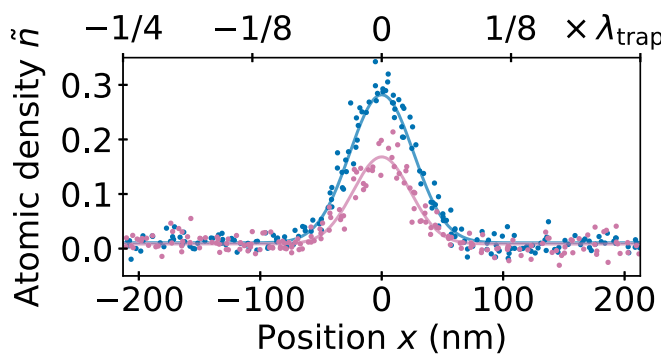

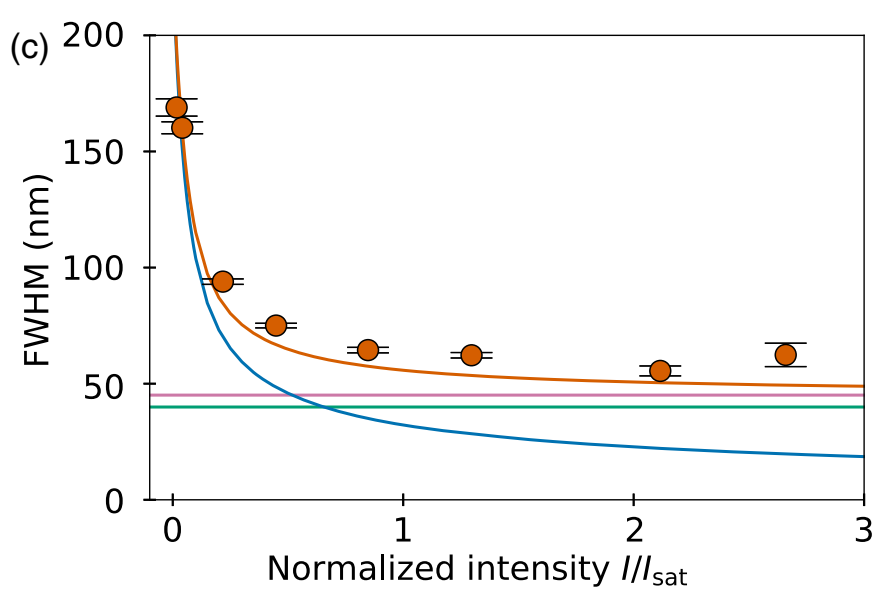

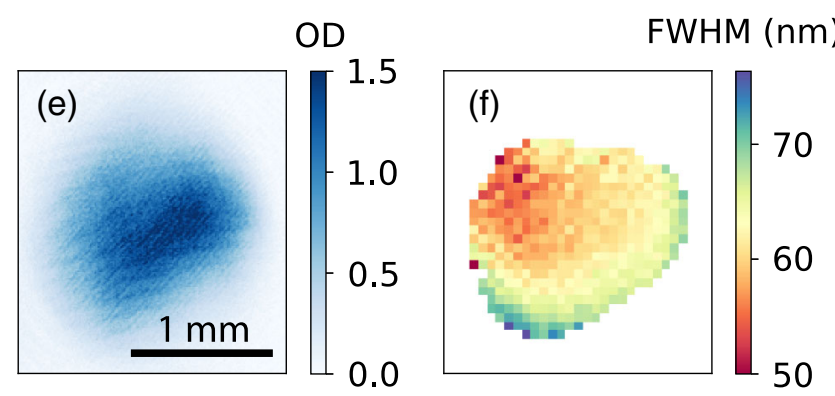

FIG. 2. Performance of superresolution imaging. (a) Images of excitation fraction $\mathcal{F}$ taken at different piezoelectric displacements $\Delta x$. For these images, the OP intensity is $I / I_{\text {sat }}=1.3$, and atoms are prepared in a trapping lattice with detuning $\delta=10 \mathrm{GHz}$ and lattice depth $U=200 \mu \mathrm{K}$. The region outlined in green indicates the area from which the data in panels (b)-(d) are collected. (b) As the optical pumping intensity increases, the atom response becomes more nonlinear, and the dips in the excitation fraction narrow. These dips reflect the atomic density distribution in each lattice site. From low to high, the traces show measurements at increasing intensity with $I / I_{\text {sat }}=0.017$ (light blue), 0.041 (green), 0.22 (orange), 1.3 (blue), 2.1 (purple), and 2.7 (gray). Solid curves show fits based on a sum of Gaussians separated by $\lambda_{\mathrm{OP}} / 2$. Piezoelectric displacement is interferometrically measured with nanometer accuracy [31]. (c) FWHMs of the fitted Gaussians at different OP intensities (orange circles) are compared with those of the ground state (40 nm, green), a thermal state with $90 \%$ ground-state occupation (45 nm, purple), theoretical resolution (blue), and the expected width (orange) of the convolution of the thermal state and the point-spread function $g(x)$ [31]. Error bars show 1 standard error. (d) Derived single-site atomic density distribution. The measurement reflects the density distribution averaged over sites contained within the green-outlined region in panel (a). From Gaussian fits, we determine FWHM = 55(2) and 62(1) nm, and uncertainty in the peak positions of 0.8 and $0.4 \mathrm{~nm}$, for $I / I_{\text {sat }}=2.1$ (purple) and 1.3 (blue), respectively. (e) A typical optical density (OD) image of all atoms. (f) Distribution of the FWHM across the cloud at $I / I_{\text {sat }}=2.1$. Only the area containing signal sufficient for fitting is shown.

distribution $\tilde{n}(\Delta x)$ is an average over sites contained in the resolution-limited spot.

For an OP pulse of duration $t \gg 1 / \Gamma$, the imaging resolution is defined based on the full width at half maximum (FWHM) $w$ of the point-spread function $g(x)$ and is calculated to be [31]

$$
w=\frac{\lambda_{\mathrm{OP}}}{2 \pi} \sqrt{\frac{2 \ln 2}{s t \beta \Gamma}},
$$

where $s=I / I_{\text {sat }}$ and $I$ is the single-beam intensity. In our experiment, the calculated imaging resolution above $s=0.6$ is high enough to reveal the shape of our atomic density distribution [see Fig. 2(c)].

Our measured widths, which reach a minimum of 55(2) $\mathrm{nm}$, are in good agreement with the expected widths from the theory prediction [see Figs. 2(c) and 2(d)]. From the measurement at $s=2.1$, we calculate an imaging resolution defined by the FWHM of the extracted point-spread function [23] to be 32(4) $\mathrm{nm}$, which is less than $1 / 25$ of the 852-nm imaging wavelength. Furthermore, from Gaussian fits, the center positions of the atomic density can be localized to about $0.4 \mathrm{~nm}$. Notably, the imaging resolution worsens at very high OP intensity $s>2.5$ because of the limited signal-to-noise ratio.

Since the extraction of the atomic density can be realized locally, we can characterize its spatial inhomogeneity across the cloud. Figure 2(f) shows the fitted widths of the measured density distributions. Here, a variation of $40 \%$ is seen, likely due to the combination of inhomogeneous cooling efficiency and trap depth. We note that such spatially resolved information about trap parameters is often inaccessible using conventional imaging techniques. 
(a)

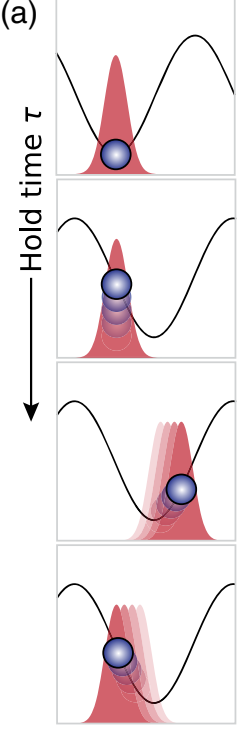

(b)

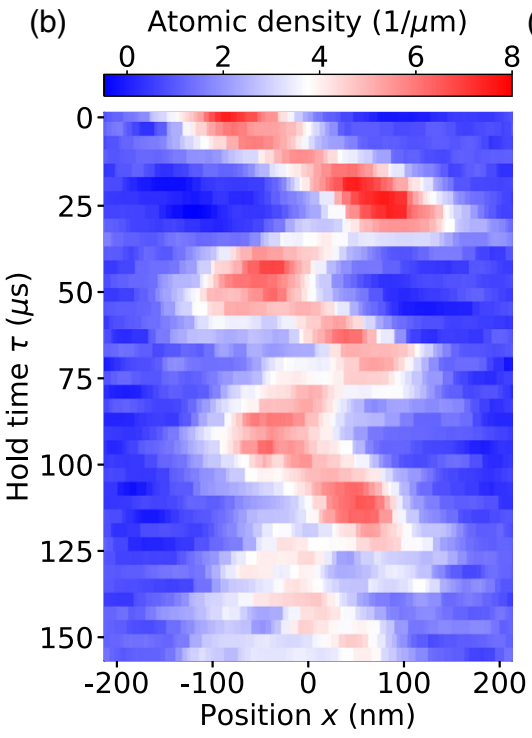

(c)
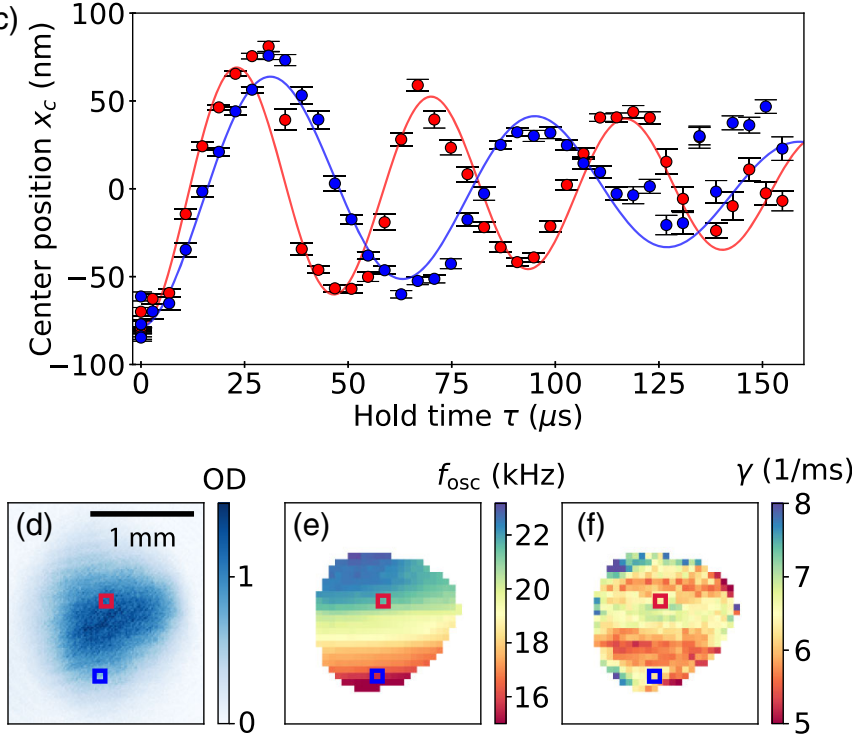

FIG. 3. Microscopic dynamics revealed by superresolution microscopy. (a) An illustration of the experimental procedure. After preparing atoms in the ground state, the trapping lattice is displaced by an amplitude $A=79 \mathrm{~nm}$, causing the atoms to oscillate. (b) Atomic density evolution within a single lattice site. After a hold time $\tau$, we record the atomic density using the same procedure as Fig. 2(d). Here, the trapping lattice detuning is $\delta=20 \mathrm{GHz}$, lattice depth $U=10 \mu \mathrm{K}$, and OP intensity $I / I_{\text {sat }}=1.3$. Jagged motion of the atoms results from anharmonicity of the lattice potential and can be described by the quantum motion in the lattice [31]. Data are extracted from the location shown in the red box in panel (d). The data presented in (b) are smoothed using a window of $\lambda_{\text {trap }} / 20$ [31]. (c) Evolution of the atomic position determined from Gaussian fits. Blue and red circles are based on measured data from bins of the same color in panel (d). Solid curves show exponentially decaying cosine fits $x_{c}=-A e^{-\gamma \tau} \cos 2 \pi f_{\text {osc }} \tau$, where $\gamma$ is the decay constant and $f_{\text {osc }}$ is the oscillation frequency. Error bars show 1 standard error. (d) Typical absorption image of the atomic cloud. (e) Map of fitted oscillation frequency $f_{\text {osc }}$ across the cloud. (f) Map of fitted decay constant $\gamma$ across the cloud.

\section{B. Motional Dynamics}

An important feature of this imaging scheme is the short microsecond duration of the OP pulse compared to the timescale of typical atomic motion in the lattice. Our scheme is thus ideally suited for probing dynamics of atoms within a lattice site. To explore this capability, we quickly displace the trapping lattice by $79 \mathrm{~nm}$ and record the evolution of the atomic density distribution after different hold times (see Fig. 3).

The displacement initiates an oscillatory motion of the atoms [see Fig. 3(b)]. The "jagged" features of the motion come from the anhamonicity of the lattice potential [31]. From the time evolution, we further extract the oscillation frequency and damping rate of the atomic motion, as shown in Fig. 3(c) for two bins in separate locations, and construct the complete maps of these quantities in the sample [see Figs. 3(d) and 3(e)], which clearly show the inhomogeneity of lattice parameters.

\section{Moiré Magnification}

Thus far, all measurements have required repeating the experiment many times, each with a small increment in the piezoelectric displacement. Here, we develop an alternative method that exploits the slight difference in wavelengths of the optical pumping and trapping beams to obtain the atomic density distribution at nanometer scale in a single shot based on the moiré effect.

When two gratings of slightly different periodicity overlap, a moiré interference pattern emerges at a macroscopic length scale [see Fig. 4(a) for an example] because the relative phase of the two gratings advances slowly and linearly along the grating direction.

In our experiment, the slight difference in the wavelengths of the trapping lattice $\lambda_{\text {trap }}$ and OP lattice $\lambda_{\mathrm{OP}}$ causes the atoms trapped in neighboring lattice sites to be probed at slightly different positions within each site. If the atomic density profile is identical along the lattice direction, the resulting moiré pattern imprinted onto the cloud represents a greatly magnified image of the density profile [see Fig. 4(b)]. The magnification $M$ is given by [32]

$$
M=\frac{\lambda_{\mathrm{OP}}}{\left|\lambda_{\mathrm{OP}}-\lambda_{\text {trap }}\right|},
$$

which in our experiment can expand 10-nm features to 1 -mm scale in a single-shot image.

Figure 4(c) shows a representative series of moiré patterns of excitation fractions observed at different detunings of the trapping lattice. The stripes appearing with greater number at larger detuning show the rephasing 


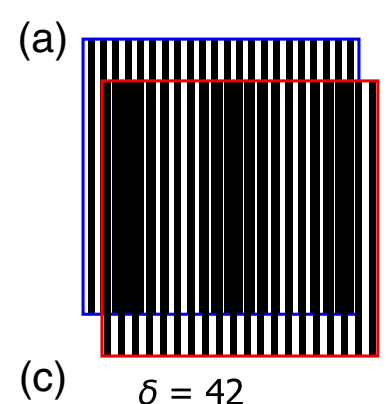

(b)

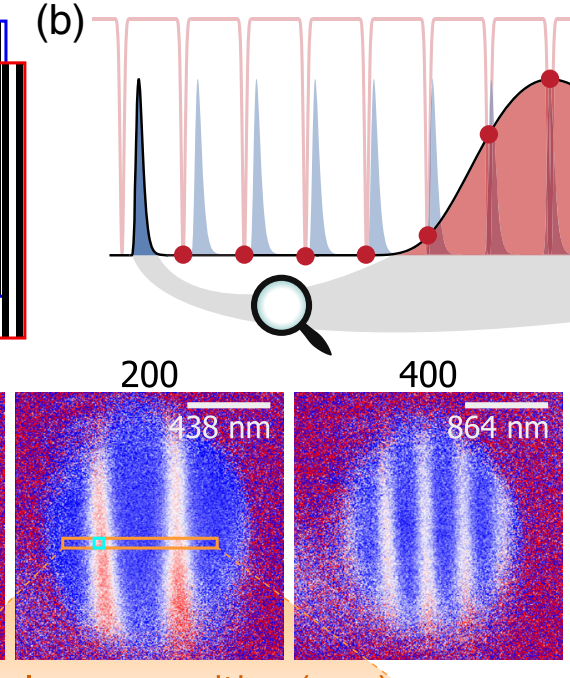

Real space position $(\mathrm{mm})$

(d)



(e)

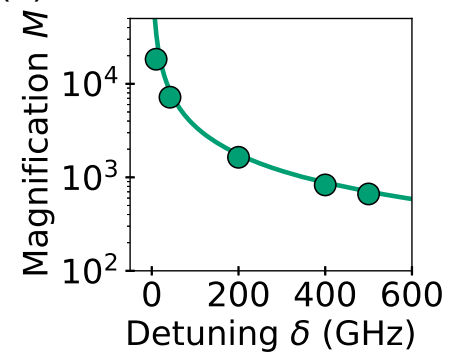

(f) $1-\mathcal{F}$
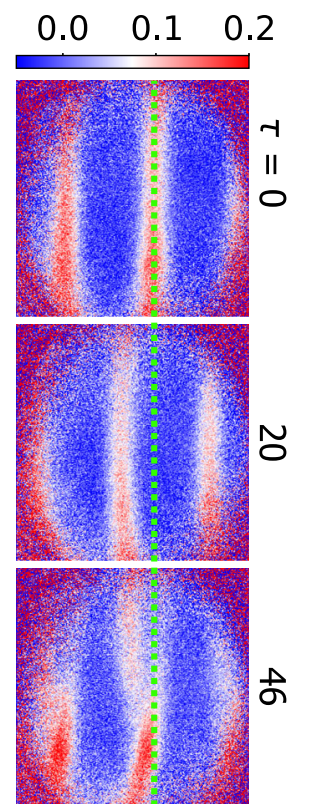

मे

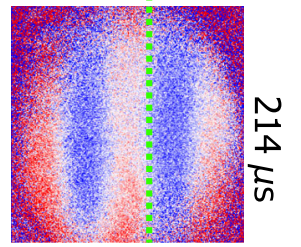

FIG. 4. Moiré magnification of the atomic density distribution. (a) When two periodic structures of slightly different pitch are overlapped, a large-scale moiré pattern emerges. (b) Magnification of the atomic density distribution based on moiré interference. The OP lattice (red) interrogates the atomic density distribution (blue shaded) at different positions (red dots) within each confining lattice site. If the atomic density distribution in every site is identical, then the resulting excitation fraction across the cloud traces out the density distribution with large magnification. (c) In situ images of excitation fraction taken at different lattice detunings $\delta$ with a field of view of $(2.44 \mathrm{~mm})^{2}$ with $I / I_{\text {sat }}=0.89$. In each image, the moiré pattern reflects the microscopic atomic density distribution. Spacing between stripes is $\lambda_{\text {trap }} / 2$. White bars show the microscopic length scale. (d) Comparison of the moiré pattern within the orange rectangle in panel (c) (orange circles) to the microscopic atomic density distribution measured in the cyan box in panel (c) by piezoelectric scan (cyan circles). The horizontal axis of each dataset is scaled to match spatial periodicity and translated to overlap the peaks. (e) Dependence of moiré magnification $M$ on detuning $\delta$ [31]. The solid line is based on Eq. (4). The statistical errors are smaller than the marker size. (f) Evolution of the moiré pattern after a 79-nm lattice phase jump with detuning $\delta=200 \mathrm{GHz}$. After hold time $\tau$, the moiré pattern oscillates and becomes distorted, indicating that the dynamics of the atoms in the lattice are not uniform across the sample. The dashed green line serves as a reference indicating the initial position of the center stripe.

of the two lattices, and the separation between two stripes corresponds to the microscopic lattice constant.

To confirm that the moiré patterns represent a faithful magnification of the atomic density distribution in a lattice site, we compare the pattern to the density profile extracted from piezoelectric scanning [see Fig. 4(d)]. Here, a weaker lattice is chosen so that the measured width is dominated by that of the atoms. The two measurements match excellently, which confirms the interpretation of a moiré pattern as a magnified image of atomic density distribution in a lattice site. We determine the magnification for each image in Fig. 4(c), and the result also shows good agreement with Eq. (4). At the smallest detuning of $10 \mathrm{GHz}$ in our experiment, the magnification reaches $M=20000$.
This moiré-pattern-based imaging scheme is also a convenient tool to study the atomic dynamics in the lattice. After displacing the lattice by $79 \mathrm{~nm}$, the moiré pattern appears straight in the beginning but develops snaking wiggles after 20-30 $\mu$ s and finally relaxes to a wider stripe at a displaced location [see Fig. 4(f)]. The snaking wiggles in each stripe indicate the inhomogeneous trap parameters across the cloud, confirming the observation in Fig. 3(c) based on piezoelectric tuning.

\section{CONCLUSION}

Our imaging method is generic and can be readily applied to other atoms and molecules. Extension of the method to two and three dimensions is straightforward. By 
incorporating this scheme in a system with high-fidelity state preparation and single-site imaging resolution (e.g., quantum gas microscopes), one can gain full spatial information of the quantum ensemble down to the nanometer scale.

The ability to resolve atomic distributions within single sites will be a powerful tool for quantum simulation of lattice models with multiple orbital bands [33,34], analyzing lattice gases with high on-site occupancy number [35-37], and characterizing pairing in the Bose-Einstein condensation-Bardeen-Cooper-Schrieffer regime in attractive Fermi-Hubbard models [38-40]. Our scheme is also well suited for studying atoms in driven optical lattices [41]. The fast imaging timescale of microsecond and spatial resolution of nanometers will enable the observation of atomic micromotion in the lattice, essential to the understanding of heating, parametric instability, and the synthesis of artificial gauge fields [2,42-44].

Our scheme also suggests convenient alternatives for realizing site-resolved microscopy when the lattice constant is well below the imaging resolution. For example, if $\lambda_{\text {trap }} / \lambda_{\mathrm{OP}}$ is a noninteger rational number, only atoms spaced by several sites will be simultaneously imaged. Combining several images at different OP lattice phases can then reconstruct occupancies at every site. Such a scheme is similar to structured illumination microscopy [24].

We thank G. Downs, P. Ocola, and M. Usatyuk for assistance in the construction of the experiment, and L.W. Clark for carefully reading the manuscript. M. M. is supported by the University of Chicago PSD Dean's postdoc fellowship. This work is supported by the Army Research Office under Grant No. W911NF-15-1-0113 and partially supported by the University of Chicago Materials Research Science and Engineering Center, which is funded by the National Science Foundation under Grant No. DMR-1420709.

Note added in proof.-Recently, we became aware of similar work from Trey Porto's group [45].

[1] N. Gemelke, X. Zhang, C.-L. Hung, and C. Chin, In Situ Observation of Incompressible Mott-Insulating Domains in Ultracold Atomic Gases, Nature (London) 460, 995 (2009).

[2] C. V. Parker, L.-C. Ha, and C. Chin, Direct Observation of Effective Ferromagnetic Domains of Cold Atoms in a Shaken Optical Lattice, Nat. Phys. 9, 769 (2013).

[3] M. Endres, M. Cheneau, T. Fukuhara, C. Weitenberg, P. Schauß, C. Gross, L. Mazza, M. C. Banuls, L. Pollet, I. Bloch et al., Observation of Correlated Particle-Hole Pairs and String Order in Low-Dimensional Mott Insulators, Science 334, 200 (2011).

[4] M. Cheneau, P. Barmettler, D. Poletti, M. Endres, P. Schauß, T. Fukuhara, C. Gross, I. Bloch, C. Kollath, and S. Kuhr,
Light-Cone-Like Spreading of Correlations in a Quantum Many-Body System, Nature (London) 481, 484 (2012).

[5] C.-L. Hung, V. Gurarie, and C. Chin, From Cosmology to Cold Atoms: Observation of Sakharov Oscillations in a Quenched Atomic Superfluid, Science 341, 1213 (2013).

[6] J.-P. Brantut, J. Meineke, D. Stadler, S. Krinner, and T. Esslinger, Conduction of Ultracold Fermions through a Mesoscopic Channel, Science 337, 1069 (2012).

[7] A. M. Kaufman, B. J. Lester, C. M. Reynolds, M. L. Wall, M. Foss-Feig, K. R. A. Hazzard, A. M. Rey, and C. A. Regal, Two-Particle Quantum Interference in TunnelCoupled Optical Tweezers, Science 345, 306 (2014).

[8] R. Blatt and D. Wineland, Entangled States of Trapped Atomic Ions, Nature (London) 453, 1008 (2008).

[9] K. D. Nelson, X. Li, and D. S. Weiss, Imaging Single Atoms in a Three-Dimensional Array, Nat. Phys. 3, 556 (2007).

[10] M. Saffman, T. G. Walker, and K. Mølmer, Quantum Information with Rydberg Atoms, Rev. Mod. Phys. 82, 2313 (2010).

[11] W. S. Bakr, J. I. Gillen, A. Peng, S. Fölling, and M. Greiner, A Quantum Gas Microscope for Detecting Single Atoms in a Hubbard-Regime Optical Lattice, Nature (London) 462, 74 (2009).

[12] J. F. Sherson, C. Weitenberg, M. Endres, M. Cheneau, I. Bloch, and S. Kuhr, Single-Atom-Resolved Fluorescence Imaging of an Atomic Mott Insulator, Nature (London) 467, 68 (2010).

[13] A. Omran, M. Boll, T. A. Hilker, K. Kleinlein, G. Salomon, I. Bloch, and C. Gross, Microscopic Observation of Pauli Blocking in Degenerate Fermionic Lattice Gases, Phys. Rev. Lett. 115, 263001 (2015).

[14] L. W. Cheuk, M. A. Nichols, M. Okan, T. Gersdorf, V. V. Ramasesh, W. S. Bakr, T. Lompe, and M. W. Zwierlein, Quantum-Gas Microscope for Fermionic Atoms, Phys. Rev. Lett. 114, 193001 (2015).

[15] M. F. Parsons, F. Huber, A. Mazurenko, C. S. Chiu, W. Setiawan, K. Wooley-Brown, S. Blatt, and M. Greiner, SiteResolved Imaging of Fermionic Li 6 in an Optical Lattice, Phys. Rev. Lett. 114, 213002 (2015).

[16] G. J. A. Edge, R. Anderson, D. Jervis, D. C. McKay, R. Day, S. Trotzky, and J. H. Thywissen, Imaging and Addressing of Individual Fermionic Atoms in an Optical Lattice, Phys. Rev. A 92, 063406 (2015).

[17] R. Yamamoto, J. Kobayashi, T. Kuno, K. Kato, and Y. Takahashi, An Ytterbium Quantum Gas Microscope with Narrow-Line Laser Cooling, New J. Phys. 18, 023016 (2016).

[18] C. W. McCutchen, Superresolution in Microscopy and the Abbe Resolution Limit, J. Opt. Soc. Am. 57, 1190 (1967).

[19] T. Gericke, P. Würtz, D. Reitz, T. Langen, and H. Ott, HighResolution Scanning Electron Microscopy of an Ultracold Quantum Gas, Nat. Phys. 4, 949 (2008).

[20] J. D. Wong-Campos, K. G. Johnson, B. Neyenhuis, J. Mizrahi, and C. Monroe, High-Resolution Adaptive Imaging of a Single Atom, Nat. Photonics 10, 606 (2016).

[21] A. Alberti, C. Robens, W. Alt, S. Brakhane, M. Karski, R. Reimann, A. Widera, and D. Meschede, Super-Resolution Microscopy of Single Atoms in Optical Lattices, New J. Phys. 18, 053010 (2016). 
[22] E. Betzig, G. H. Patterson, R. Sougrat, O. W. Lindwasser, S. Olenych, J. S. Bonifacino, M. W. Davidson, J. LippincottSchwartz, and H. F. Hess, Imaging Intracellular Fluorescent Proteins at Nanometer Resolution, Science 313, 1642 (2006).

[23] S. W. Hell and J. Wichmann, Breaking the Diffraction Resolution Limit by Stimulated Emission: StimulatedEmission-Depletion Fluorescence Microscopy, Opt. Lett. 19, 780 (1994).

[24] M. G. L. Gustafsson, Nonlinear Structured-Illumination Microscopy: Wide-Field Fluorescence Imaging with Theoretically Unlimited Resolution, Proc. Natl. Acad. Sci. U.S.A. 102, 13081 (2005).

[25] A. V. Gorshkov, L. Jiang, M. Greiner, P. Zoller, and M. D. Lukin, Coherent Quantum Optical Control with Subwavelength Resolution, Phys. Rev. Lett. 100, 093005 (2008).

[26] J. Cho, Addressing Individual Atoms in Optical Lattices with Standing-Wave Driving Fields, Phys. Rev. Lett. 99, 020502 (2007).

[27] G. S. Agarwal and K. T. Kapale, Subwavelength Atom Localization via Coherent Population Trapping, J. Phys. B 39, 3437 (2006).

[28] E. Paspalakis and P. L. Knight, Localizing an Atom via Quantum Interference, Phys. Rev. A 63, 065802 (2001).

[29] J. A. Miles, Z. J. Simmons, and D. D. Yavuz, Subwavelength Localization of Atomic Excitation Using Electromagnetically Induced Transparency, Phys. Rev. X 3, 031014 (2013).

[30] A. J. Kerman, V. Vuletić, C. Chin, and S. Chu, Beyond Optical Molasses: $3 d$ Raman Sideband Cooling of Atomic Cesium to High Phase-Space Density, Phys. Rev. Lett. 84, 439 (2000).

[31] See Supplemental Material at http://link.aps.org/ supplemental/10.1103/PhysRevX.9.021001 for more detailed discussions.

[32] S. Morse, A. J. Durelli, and C. A. Sciammarella, Geometry of Moiré Fringes in Strain Analysis, J. Eng. Mech. 86, 105 (1960).

[33] M. Anderlini, P. J. Lee, B. L. Brown, J. Sebby-Strabley, W. D. Phillips, and J. V. Porto, Controlled Exchange Interaction between Pairs of Neutral Atoms in an Optical Lattice, Nature (London) 448, 452 (2007).

[34] T. Müller, S. Fölling, A. Widera, and I. Bloch, State Preparation and Dynamics of Ultracold Atoms in Higher Lattice Orbitals, Phys. Rev. Lett. 99, 200405 (2007).
[35] G. K. Campbell, J. Mun, M. Boyd, P. Medley, A. E. Leanhardt, L. G. Marcassa, D. E. Pritchard, and W. Ketterle, Imaging the Mott Insulator Shells by Using Atomic Clock Shifts, Science 313, 649 (2006).

[36] S. Will, T. Best, U. Schneider, L. Hackermüller, D.-S. Lühmann, and I. Bloch, Time-Resolved Observation of Coherent Multi-Body Interactions in Quantum Phase Revivals, Nature (London) 465, 197 (2010).

[37] A. Goban, R. B. Hutson, G. E. Marti, S. L. Campbell, M. A. Perlin, P. S. Julienne, J. P. D'Incao, A. M. Rey, and J. Ye, Emergence of Multi-Body Interactions in a Fermionic Lattice Clock, Nature (London) 563, 369 (2018).

[38] C. H. Schunck, Y. Shin, A. Schirotzek, M. W. Zwierlein, and W. Ketterle, Pairing without Superfluidity: The Ground State of an Imbalanced Fermi Mixture, Science 316, 867 (2007).

[39] T. Esslinger, Fermi-Hubbard Physics with Atoms in an Optical Lattice, Annu. Rev. Condens. Matter Phys. 1, 129 (2010).

[40] D. Mitra, P. T. Brown, E. Guardado-Sanchez, S. S. Kondov, T. Devakul, D. A. Huse, P. Schauss, and W. S. Bakr, Quantum Gas Microscopy of an Attractive Fermi-Hubbard System, Nat. Phys. 14, 173 (2018).

[41] A. Eckardt, Colloquium: Atomic Quantum Gases in Periodically Driven Optical Lattices, Rev. Mod. Phys. 89, 011004 (2017).

[42] J. Struck, C. Ölschläger, M. Weinberg, P. Hauke, J. Simonet, A. Eckardt, M. Lewenstein, K. Sengstock, and P. Windpassinger, Tunable Gauge Potential for Neutral and Spinless Particles in Driven Optical Lattices, Phys. Rev. Lett. 108, 225304 (2012).

[43] M. Weinberg, C. Ölschläger, C. Sträter, S. Prelle, A. Eckardt, K. Sengstock, and J. Simonet, Multiphoton Interband Excitations of Quantum Gases in Driven Optical Lattices, Phys. Rev. A 92, 043621 (2015).

[44] L. W. Clark, B. M. Anderson, L. Feng, A. Gaj, K. Levin, and C. Chin, Observation of Density-Dependent Gauge Fields in a Bose-Einstein Condensate Based on Micromotion Control in a Shaken Two-Dimensional Lattice, Phys. Rev. Lett. 121, 030402 (2018).

[45] S. Subhankar, Y. Wang, T-C. Tsui, S. L. Rolston, and J. V. Porto, following paper, Nanoscale Atomic Density Microscopy, Phys. Rev. X 9, 021002 (2018). 